\title{
MODELLING OF THE FLOW OF STREAMS OF COHESIONLESS AND COHESIVE BULK MATERIALS IN A CONVEYOR DISCHARGE POINT WITH A FLAT CONVEYOR BELT
}

\author{
J.A. CYGANIUK* ${ }^{*}$ and P. KURYŁO \\ University of Zielona Góra, Faculty of Mechanical Engineering \\ Institute of Mechanical Engineering and Machine Operation \\ ul. Szafrana 4, 65-516 Zielona Góra, POLAND \\ E-mails: J.Cyganiuk@ibem.uz.zgora.pl \\ P.Kurylo@ibem.uz.zgora.pl
}

\begin{abstract}
The paper presents the analysis of flow conditions of cohesive and cohesionless bulk materials in a conveyor discharge point of a flat conveyor belt. The analysis was carried out for stationary flows at high velocities. It presents mathematical methods for the description of the velocity of a material leaving a throwing point of a flat conveyor belt as well as final equations which enable the determination of velocity of the material after it has left the throwing point (with the accuracy sufficient for practical use). Next, the velocity calculated for the proposed mathematical description (for selected material groups) has been compared with the velocity obtained from mathematical relations commonly used by engineers. The proposed equations for determining the velocity of the material beyond the point have proved useful, since they enable excluding the indirect equations. Finally, the difference between the values of the velocity obtained with the proposed and indirect equations have been determined and the relative error for the proposed method has been calculated.
\end{abstract}

Key words: pouring points, discharge point, bulk materials, adhesion, modelling of the flow, material velocity.

\section{Introduction}

In transport conveyor systems built on the basis of belt conveyors, pouring points are important parts. They are crucial elements allowing transporting bulk materials on different levels of this type of system. An important issue in a conveyor transport is to ensure a proper flow of the material through pouring points. This involves ensuring continuity of the transported material stream and ensuring the required transport capacity of the system. Pouring points used in transport systems often exploit gravity to transport materials. These types of pouring points include, e.g. chutes described in Antoniak [1] and Żur [2]. Another type of pouring points that cooperate with belt conveyors are impact plates described in Cyganiuk [3, 4, 5], Cyganiuk and Przystupa [6], Korzeń [7], Przystupa and Cyganiuk [8], Sakowich and Kuksa [9]. They are used to slow down the velocity of the material to a value corresponding with the velocity of the receiving conveyor in the feed point.

A throwing point with a flat conveyor belt, discussed further, uses a driven belt for moving and throwing the material. However, the point is not inclined as described in Cyganiuk and Przystupa [10], Cyganiuk [11], Cyganiuk et al. [12], Korzeń [13]. This means that the angle of inclination of the conveyor belt is $0^{\circ}$ (Korzeń $[7,14]$ ).

In transport systems, slow belt conveyors are most commonly used. They operate with velocities below $3 \mathrm{~m} / \mathrm{s}$ (described in Antoniak [1] and Żur [2]), and equations determining the velocity of cohesive and cohesionless materials beyond the conveyor pulley are described in Korzeń [13]. However, these equations should not be used for fast conveyors. Equations for fast conveyors are presented also in Korzeń [13]. They

\footnotetext{
* To whom correspondence should be addressed
} 
do not take adhesion into consideration, but include the influence of air resistance on the velocity of the transported material beyond the head pulley. In Korzen [14], the influence of adhesion on the velocity of the material beyond the point was also omitted. However, the study included material, kinematic and dynamic parameters. In Colijn [15], the adhesion phenomenon also was omitted.

In the case of discharge belt conveyors (Korzen [16]), the construction of this type of conveyors and the discharge method of the material are different from the ones used for a belt conveyor feeding the next pouring point. Thus, equations proposed in Korzeń [16] designed for estimating the velocity of the material beyond the pulley, should not be used for fast feeding belt conveyors.

In Czuba and Furmanik [17], only kinematic and geometric parameters for the estimation of the velocity of the material falling down onto a belt conveyor were taken into consideration, whereas Antoniak [18] discusses simple engineering equations describing the velocity of the material flowing from a belt conveyor. They take into consideration the coefficient of friction of the material against the belt but do not take into consideration forces and adhesion affecting the material stream.

Cyganiuk and Przystupa [10], Cyganiuk [11], Cyganiuk et al. [12] take into account kinematic, dynamic and material parameters including adhesion, but the equations presented there are designed for inclined conveyors with ascending and/or descending belts.

Cyganiuk in [3] analyses the trajectory of the material beyond a throwing point depending on the velocity of a belt conveyor, but they do not analyse the velocity of the material beyond the point where it reaches the next pouring point. In addition, Arnold and Hill [19] and also Hastie and Wypych [20] refers to the prediction of the trajectory of the material beyond the throwing point, but in this case, both fast and slow conveyors were taken into consideration.

Therefore, it was important to propose equations describing the velocity of the material beyond the pulley of a not inclined belt conveyor, equipped with the belt without cleats for both cohesive and cohesionless materials.

\section{Analysis of flow conditions of cohesionless and cohesive materials in the throwing point with a flat belt}

In conveyor transport systems, a throwing point refers to a head pulley of the belt conveyor. From the pulley, the material is transferred further, as a feed, onto another conveyor, which, most often, is located lower and the feed is delivered with the use of a chute (Antoniak [1], Żur [2]) or an impact plate (Antoniak [1], Cyganiuk [11, 3, 4, 5], Cyganiuk and Przystupa, [6], Korzeń [7], Żur [2]). The material from the throwing point may also be thrown directly onto hillocks.

The analysis of flow conditions of cohesive and cohesionless bulk materials in a throwing point with a flat belt is shown in Figs 1 and 2. The analysis was carried out both for fast conveyors, where the belt transports the material with the velocity above $3[\mathrm{~m} / \mathrm{s}]$, and for stationary flow. A belt without cleats was analysed and the influence of air resistance was not considered.

Figures 1 and 2 illustrate magnitudes taken into consideration in the analysis of the flow conditions that affect the process of discharging the material from the conveyor's pulley. Geometric, kinematic and dynamic conditions of the motion of elementary mass $\mathrm{dm}$ in a throwing point, which is not inclined, were taken into consideration.

In a throwing point where cohesionless materials are discharged, the elementary mass $\mathrm{dm}$ is subject to the following forces describing dynamic conditions of flow (Fig.1): gravitational force $d \boldsymbol{G}[N]$, centrifugal force $d \boldsymbol{F}_{c}[N]$, normal force $d \boldsymbol{N}_{x s}[N]$, tangential force $d \boldsymbol{T}_{x s}[N]$. While determining the relations describing the discharge of the material from the pulley, the inertial force $d J[N]$ formed as a result of action of the gravitational force $d \boldsymbol{G}$, centrifugal force $d \boldsymbol{F}_{c}$ and forces $d \boldsymbol{N}_{x s}, d \boldsymbol{T}_{x s}$ was also considered.

In the case of cohesive materials, the dynamic conditions of the flow of the material in the throwing point, besides the above mentioned forces, also adhesion force $d \boldsymbol{F}_{a}$ (Fig.2) was considered. The adhesion force forms between the belt and the transported material.

Kinematic conditions of the flow of material in a throwing point with a flat belt (Figs 1 and 2) include: the velocity of the conveyor belt $v_{t}[\mathrm{~m} / \mathrm{s}]$, the velocity of bulk material stream flowing into the head 
pulley $v_{p}[\mathrm{~m} / \mathrm{s}]$, which is equal to the velocity of the belt, and the velocity of material leaving the head pulley $v_{w}[\mathrm{~m} / \mathrm{s}]$.

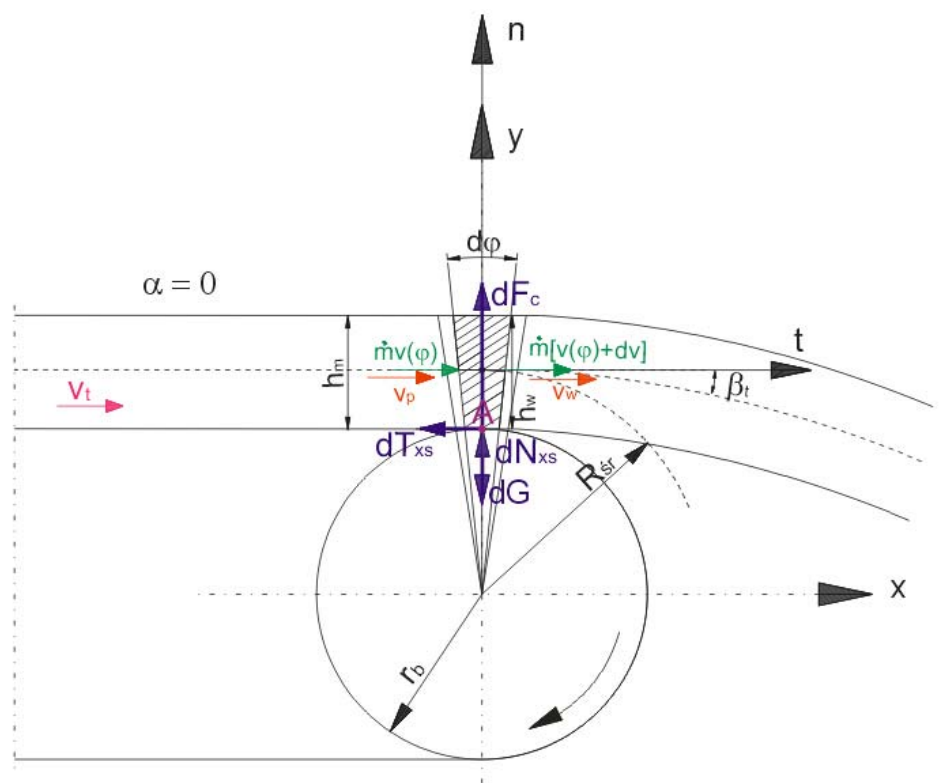

Fig.1. Geometric, kinematic and dynamic conditions of the flow of a stream of a cohesionless bulk material on a head pulley of a conveyor with a flat belt [own elaboration].

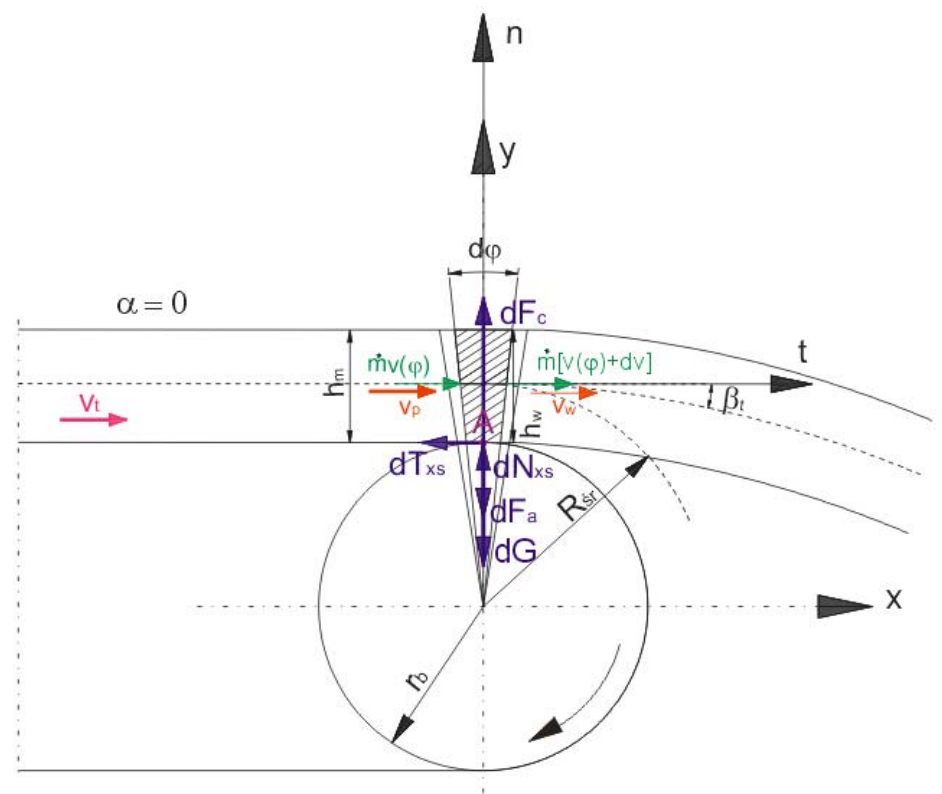

Fig.2. Geometric, kinematic and dynamic conditions of the flow of a stream of cohesive bulk material on a head pulley of a conveyor with a flat belt (Cyganiuk [3]).

Geometric conditions of the material flowing out from the throwing point include the following parameters (Cyganiuk et al. [12]): the angle of conveyor descent $\alpha\left[^{\circ}\right]$ (in this case $\alpha=0^{\circ}$ ), angle of the material stream flowing out of the head pulley $\beta_{t}\left[{ }^{\circ}\right]$ (in this case $\beta_{t}$ is the angle of material flowing into another point, e.g. impact point), angle coordinate which describes the position of infinitesimal mass of the material on the head pulley $\varphi\left[{ }^{\circ}\right]$ (Cyganiuk et al.[12]), radius of the head pulley $r_{b}[m]$, the average radius of 
curvature of the material stream $R_{s r}[m]$, thickness of the layer of the material stream at the moment it flows onto the head pulley $h_{m}[m]$ (Cyganiuk et al. [12]), thickness of the layer of the material stream leaving the head pulley $h_{w}[\mathrm{~m}]$ (Cyganiuk et al. [12]).

Kinematic and geometric conditions of the material flowing out of a head pulley with a flat belt for cohesive and cohesionless materials are the same, whereas in the case of dynamic conditions - the difference is the adhesion force that appears in the case of cohesive materials.

\section{Mathematical model of the flow of a stream of a cohesionless and cohesive bulk material in a throwing point with a flat belt}

In the analysis of the discharge of the material from a throwing point with a flat belt, it was assumed that the material is separated from the pulley at point A (Figs 1 and 2). This is the point in which the belt overlaps the head pulley. Regardless of the type of transported material, Eq.(3.1) must be satisfied to let the material leave the belt (Antoniak [1])

$$
\frac{v^{2}}{R}>g \cdot \cos \alpha
$$

where: $v$ - belt velocity $[\mathrm{m} / \mathrm{s}], R$ - radius of the pulley $[\mathrm{m}], \mathrm{g}$ - gravitational acceleration $\left[\mathrm{m} / \mathrm{s}^{2}\right], \alpha$ - the angle of the conveyor descend $\left[^{\circ}\right]$, (Antoniak [1],Cyganiuk et al.[12]).

For cohesive materials, also Eq.(3.2), taking into consideration adhesion and high velocities of the conveyor, must be satisfied (Korzeń [13])

$$
\frac{F_{r}-A_{d}}{\cos \alpha} \geq 1
$$

where

$$
\begin{gathered}
A_{d}=\frac{\sigma_{a}}{\gamma \cdot h_{m}}, \\
F_{r}=\frac{v^{2}}{g \cdot R}
\end{gathered}
$$

where: $v$ - belt velocity $[\mathrm{m} / \mathrm{s}], \gamma$ - specific gravity of bulk material $\left[\mathrm{N} / \mathrm{m}^{3}\right], \sigma_{a}$ - adhesion $\left[\mathrm{N} / \mathrm{m}^{2}\right], \mathrm{hm}$ thickness of the material stream $[\mathrm{m}], \mathrm{g}$ - gravitational acceleration $\left[\mathrm{m} / \mathrm{s}^{2}\right], R$ - radius of the pulley $[\mathrm{m}]$ (Cyganiuk et al. [12]).

To obtain mathematical relations allowing determination of velocity $v(\varphi)[\mathrm{m} / \mathrm{s}]$ of a cohesive and cohesionless bulk material beyond the throwing points with a flat belt, a system of equations was used. The system differs depending on the type of material, and for a cohesionless material it includes:

- $\quad$ equation of continuity (Cyganiuk et al. [12])

$$
\dot{m}=\rho \cdot v(\varphi) \cdot A(\varphi)
$$

equation of equilibrium (Cyganiuk et al. [12])

$$
\dot{m}(\boldsymbol{v}+d \boldsymbol{v})-\dot{m} \boldsymbol{v}=d \boldsymbol{G}+d \boldsymbol{F}_{C}+d \boldsymbol{N}_{x S}+d \boldsymbol{T}_{x S}
$$


- equation describing the contact friction condition on surfaces of walls restricting the path of the flow of a bulk material stream for static conditions and cohesionless materials (according to Korzeń [13])

$$
\tau_{w S}=\sigma_{n} \mu_{x S}
$$

For cohesive materials, the system of equations includes:

equation of continuity (Cyganiuk et al. [12])

$$
\dot{m}=\rho \cdot v(\varphi) \cdot A(\varphi)
$$

- $\quad$ equation of equilibrium (Cyganiuk et al. [12])

$$
\dot{m}(\boldsymbol{v}+d \boldsymbol{v})-\dot{m} \boldsymbol{v}=d \boldsymbol{G}+d \boldsymbol{F}_{C}+d \boldsymbol{F}_{a}+d \boldsymbol{N}_{x S}+d \boldsymbol{T}_{x S}
$$

- equation describing the contact friction condition on the surfaces of walls restricting the path of the flow of a bulk material stream for static conditions and cohesive materials (according to Korzeń [13])

$$
\tau_{w s}=\left(\sigma_{n}+\sigma_{a}\right) \mu_{x s}
$$

In Eqs (3.7) and (3.9), $\mu_{x s}$ is a contact friction coefficient for static conditions. The general form of the system of equations for a cohesionless material takes the form Eq.(3.11) (Cyganiuk et al. [12])

$$
\begin{aligned}
& \dot{m}=\rho \cdot v(\varphi) \cdot A(\varphi) \\
& \dot{m}(\boldsymbol{v}+d \boldsymbol{v})-\dot{m} \boldsymbol{v}=d \boldsymbol{G}+d \boldsymbol{F}_{C}+d \boldsymbol{N}_{X S}+d \boldsymbol{T}_{X S}, \\
& \tau_{w S}=\sigma_{n} \mu_{X S}
\end{aligned}
$$

whereas for cohesive materials, the form of the system of equations is as follows (Cyganiuk et al. [12])

$$
\begin{aligned}
& \dot{m}=\rho \cdot v(\varphi) \cdot A(\varphi) \\
& \dot{m}(\boldsymbol{v}+d \boldsymbol{v})-\dot{m} \boldsymbol{v}=d \boldsymbol{G}+d \boldsymbol{F}_{C}+\boldsymbol{F}_{a}+d \boldsymbol{N}_{x S}+d \boldsymbol{T}_{x S}, \\
& \tau_{w S}=\left(\sigma_{n}+\sigma_{a}\right) \mu_{x S} .
\end{aligned}
$$

A detailed description of parameters included in the systems of Eqs (3.11) and (3.12) is the same as presented in Cyganiuk et al. [12], where $A(\varphi)$ - cross-section of the stream [ $\left.\mathrm{m}^{2}\right], \rho$ - bulk material density $\left[\mathrm{kg} / \mathrm{m}^{3}\right], \dot{\mathrm{m}}$ - mass flow $[\mathrm{kg} / \mathrm{s}]$.

Projection of Eq.(3.6) onto directions of the assumed coordinate system $\langle n, t>$ (Fig.1) will generate a system of equations as a function of the angular parameter $\varphi$, which after simplification, for cohesionless materials, takes the form 


$$
\begin{aligned}
& \mu_{x S}\left|d \boldsymbol{F}_{c}\right|-\mu_{x S}|d \boldsymbol{G}|=0, \\
& \dot{m} d v=0 .
\end{aligned}
$$

For cohesive materials projection of Eq.(3.9) onto directions of the assumed coordinate system $<n$, $t>$ (Fig.2) will also generate a system of equations as a function of the angular parameter $\varphi$, a simplified form of which is as follows

$$
\begin{aligned}
& \mu_{x S}\left|d \boldsymbol{F}_{c}\right|-\mu_{x S}\left|d \boldsymbol{F}_{a}\right|-\mu_{x S}|d \boldsymbol{G}|=0, \\
& \dot{m} d v+\mu_{x S}\left|d \boldsymbol{F}_{a}\right|=0 .
\end{aligned}
$$

The solutions to Eqs (3.13) and (3.14) are respectively differential equations, where for cohesionless materials the solution takes the following form Eq.(3.15)

$$
\frac{d v^{2}(\varphi)}{d \varphi}-2 \mu_{x S} v^{2}(\varphi)=-2 g \mu_{X S} R(\varphi)
$$

and for cohesive materials - the solution is presented by Eq.(3.16)

$$
\frac{d v^{2}(\varphi)}{d \varphi}-2 \mu_{x S} v^{2}(\varphi)=-2 \mu_{x s} g R(\varphi)\left[1+\frac{2 \sigma_{a}}{\gamma h(\varphi)}\right]
$$

Equations (3.15) and (3.16) are a special case of the Bernoulli equation, which can be written in the form (according to Korzeń [7])

$$
y^{\prime}+P(\varphi) y=Q(\varphi)
$$

By integrating Eq.(3.17), equations that allow determining the velocity of the material beyond the throwing point for cohesive and cohesionless materials were obtained. For cohesionless materials the velocity is described by Eq.(3.18)

$$
v_{w}=\sqrt{C_{w} e^{2 \mu_{x S^{\varphi}}}+g R(\varphi)},
$$

and for cohesive materials by Eq.(3.19)

$$
v_{w}=\sqrt{C_{w} e^{2 \mu_{x S^{\varphi}}}+g R(\varphi)\left[1+\frac{2 \sigma_{a}}{\gamma h(\varphi)}\right]}
$$

The description of parameters included in Eqs (3.11) and (3.12) is the same as presented in Cyganiuk et al. [12], where $C_{w}$ - integration constant, $R(\varphi)$ - an average radius of curvature of the stream [m], $\left.h \varphi\right)$ thickness of the material stream (according to Fig.2, it is the height of the material stream lying on the conveyor belt). 
Equations (3.18) and (3.19) contain integration constants, which describe Eq.(3.20) for cohesionless materials, and Eq.(3.21) for cohesive materials

$$
\begin{aligned}
& C_{w}=e^{-2 \mu_{X S^{\varphi}}}\left\{v_{p}^{2}-g R(\varphi)\right\}, \\
& C_{w}=e^{-2 \mu_{X S^{\varphi}}}\left\{v_{p}^{2}-g R(\varphi)\left[1+\frac{2 \sigma_{a}}{\gamma h(\varphi)}\right]\right\} .
\end{aligned}
$$

Equations (3.18) and (3.19) as well as 3.20 and 3.21 differ from one another by an element including adhesion.

To obtain a solution, boundary conditions, which are the same for both cohesive and cohesionless materials, were taken. Thus, the following boundary conditions, allowing the determination of the integration constant, were assumed (Cyganiuk and Przystupa [10], Cyganiuk [11])

$$
\begin{aligned}
& \varphi=\alpha \\
& R(\varphi)=R(\alpha)=R_{0}=r_{b}+0.5 h_{m}, \\
& h_{m}=\frac{\dot{m}}{\rho v_{t} B}, \\
& v(\varphi)=v(\alpha)=v_{0}=v_{t}, \\
& A(\phi)=A(\alpha)=A_{0}=\frac{\dot{m}}{v(\alpha) \rho}=\frac{\dot{m}}{v_{t} \rho}=h_{m} B .
\end{aligned}
$$

Velocity $v_{w}$ allows assuming the following boundary condition (Cyganiuk and Przystupa [10], Cyganiuk [11])

$$
\varphi=\varphi_{w}=\beta_{t} .
$$

The value of the velocity of the material beyond the throwing point is obtained by applying a simple iteration method, assuming initial conditions determined from Eqs (3.22) - (3.27).

To obtain the exact approximation of velocity $v_{w}$ of the material leaving the point, the following relation should be satisfied

$$
\left|\frac{v_{w n}-v_{w(n-1)}}{v_{w n}}\right| \leq \delta_{v_{w}[\%]}
$$

where $\delta_{v w}$ is an acceptable relative deviation of the estimated velocity $v_{w}$. It is assumed that velocity $v_{w}$ was correctly determined if the value of the deviation ranges $1 \% \div 2 \%$.

Parameter $\alpha$ is constant because the conveyor is not inclined, unlike in solutions presented in Cyganiuk and Przystupa [10], Cyganiuk [11], and Cyganiuk et al. [12]. 
Equations (3.18) and (3.19) were tested to verify if they comply with the assumption that the velocity of the material directly leaving the head pulley of the conveyor is equal to the velocity of the belt. Both equations proved to be compliant with the assumption.

Equations (3.18) and (3.19) can also be used to determine the velocity of the material beyond the throwing point, depending on the assumed angle of material inflow to the next point, e.g. an impact plate (Fig.3). In this case, it may be assumed in the proposed equations that angle $\beta_{t}$ is equal to the angle at which the material falls onto a surface (e.g. impact plate). Such an approach is a simplification, but yields results sufficient for practical use.

\section{Indirect equations}

Indirect equations allow determining the velocity of the material leaving the throwing point which cooperates with an impact point presented in Korzen [7], where they were applied for a conveyor with an ascending and descending belt. These equations can also be used for a flat conveyor. They are particularly suitable for a conveyor cooperating with an impact plate and for this application they yield the best results.

Figure 3 presents the flow conditions of the material on a conveyor with a flat belt (the conveyor is not inclined), whereas Eqs (3.29) - (3.32) allow determining the numerical values of parameters that can be seen in Fig.3. These equations are usable regardless the material.

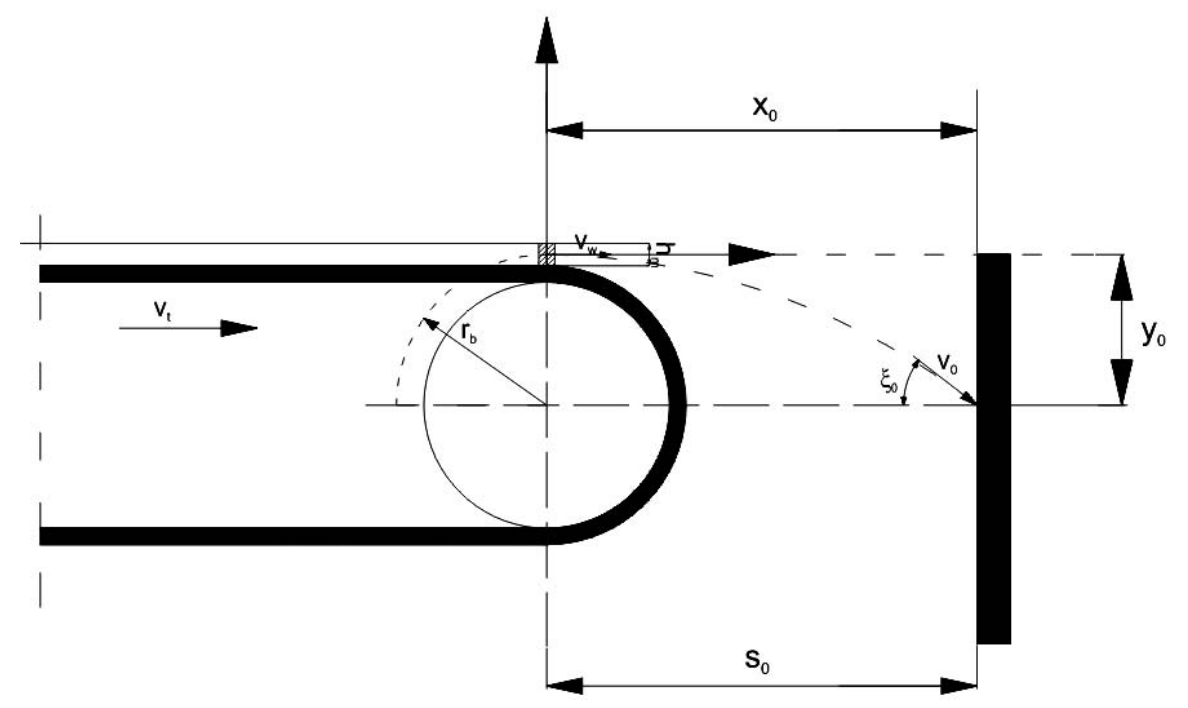

Fig.3. Cooperation of a throwing point with a flat belt and an impact point with a not inclined plate [elaborated based on Korzeń [7]].

Indirect equations (Fig.3) include (Korzeń [7]):

equation describing an angle of the material flowing onto an impact point $\xi_{0}$

$$
\xi_{0}=\operatorname{arctg}\left(\operatorname{tg} \beta_{t}-\frac{g \cdot x_{0}}{2 \cdot v_{w l}^{2} \cdot \cos ^{2} \beta_{t}}\right)
$$

equation describing the velocity of the material flowing onto an impact point $v_{0}$

$$
v_{0}=v_{w 1} \cdot \cos \beta_{t} \cdot \sqrt{\operatorname{tg}^{2} \xi_{0}+1}
$$


equations describing distance parameters, according to Fig.3

$$
\begin{aligned}
& x_{0}=s_{0}+\left(r_{b}+0.5 \cdot h_{0}\right) \cdot \sin \beta_{t}, \\
& y_{0}=\operatorname{tg} \beta_{t} \cdot x_{0}-\frac{g \cdot x_{0}^{2}}{2 \cdot v_{w_{1}} \cdot \cos ^{2} \beta_{t}}
\end{aligned}
$$

where $\beta_{t}$ is an inclination angle of the material stream leaving the conveyor. In this case, $\beta_{t}$ is equal to 0 because the conveyor is not inclined.

\section{Comparison of the calculation methods}

Table 1 was presents an example demonstrating the usefulness of the proposed equations. It also includes a comparison between the results obtained with the use of the proposed equations and the results obtained with the currently used indirect equations. In the proposed equations, the indirect equations are omitted, assuming that the angle of the outflow of the material from a head pulley is equal to the angle of the inflow of the material to the impact plate $\left(\beta_{t}=\xi_{0}\right)$. This is a considerable simplification in relation to the currently used indirect equations, where not only the velocity of the material flowing into the impact plate, but also the angle at which it flows into the plate are determined.

Table 1 presents the comparison of the results obtained with indirect equations and with the proposed equations for both cohesive and cohesionless materials. Table 1 also contains estimation of the percentage error for results yielded by the proposed equations in relation to the results obtained with the use of indirect equations. It also presents the difference between the obtained velocities of the material at the moment when it falls onto the plate. Initial conditions of the material outflow are the same for both calculation cases.

Some of the values describing the parameters of the outflow of the material from the throwing point were taken from Żur [2] and Cyganiuk et al. [12] .

Table 1. Determination of the velocity of the material beyond the discharge point in relation to the analyzed

\begin{tabular}{|c|c|}
\hline Cohesionless material & Cohesive material \\
\hline $\begin{array}{l}\text { - required mass flow } \dot{m}=350[\mathrm{~kg} / \mathrm{s}], \\
\text { - belt velocity } v_{p}=3.15[\mathrm{~m} / \mathrm{s}], \\
\text { - belt width } B_{t}=0.8[\mathrm{~m}], \\
\text { - pulley radius } r_{b}=0.25[\mathrm{~m}], \\
\text { - angle of inclination of the conveyor belt } \alpha=0\left[^{\circ}\right] \text {, } \\
\text { - gravitational acceleration } g=9.81\left[\mathrm{~m} / \mathrm{s}^{2}\right], \\
\text { - material bulk density } \rho=850\left[\mathrm{~kg} / \mathrm{m}^{3}\right], \\
\text { - specific gravity } \gamma=8340\left[\mathrm{~N} / \mathrm{m}^{3}\right], \\
\text { - friction coefficient for static conditions } \mu_{x s}=0.50, \\
\text { - angle at which bulk material flows into subsequent } \\
\text { point } \beta_{t}=\xi 0=41\left[^{\circ}\right] \text {, } \\
\text { - distance between the head pulley and the point at } \\
\text { which material is discharged } s_{0}=0.9[\mathrm{~m}],\end{array}$ & $\begin{array}{l}\text { - required mass flow } \dot{m}=350[\mathrm{~kg} / \mathrm{s}], \\
\text { - belt velocity } v_{p}=3.15[\mathrm{~m} / \mathrm{s}], \\
\text { - belt width } B_{t}=0.8[\mathrm{~m}], \\
\text { - pulley radius } r_{b}=0.25[\mathrm{~m}], \\
\text { - angle of inclination of the conveyor belt } \alpha=0\left[^{\circ}\right], \\
\text { - gravitational acceleration } g=9.81\left[\mathrm{~m} / \mathrm{s}^{2}\right], \\
\text { - material bulk density } \rho=1380\left[\mathrm{~kg} / \mathrm{m}^{3}\right], \\
\text { - specific gravity } \gamma=13540\left[\mathrm{~N} / \mathrm{m}^{3}\right], \\
\text { - friction coefficient for static conditions } \mu_{x s}=0.51, \\
\text { - angle at which bulk material flows into subsequent } \\
\text { point } \beta_{t}=\xi 0=41\left[^{\circ}\right] \text {, } \\
\text { - distance between the head pulley and the point at which } \\
\text { material is discharged } s_{0}=0.9, \\
\text { - adhesion } \sigma_{a}=350\left[\mathrm{~N} / \mathrm{m}^{2}\right],\end{array}$ \\
\hline
\end{tabular}
angle at which the material flows into the impact point [own elaboration]. 


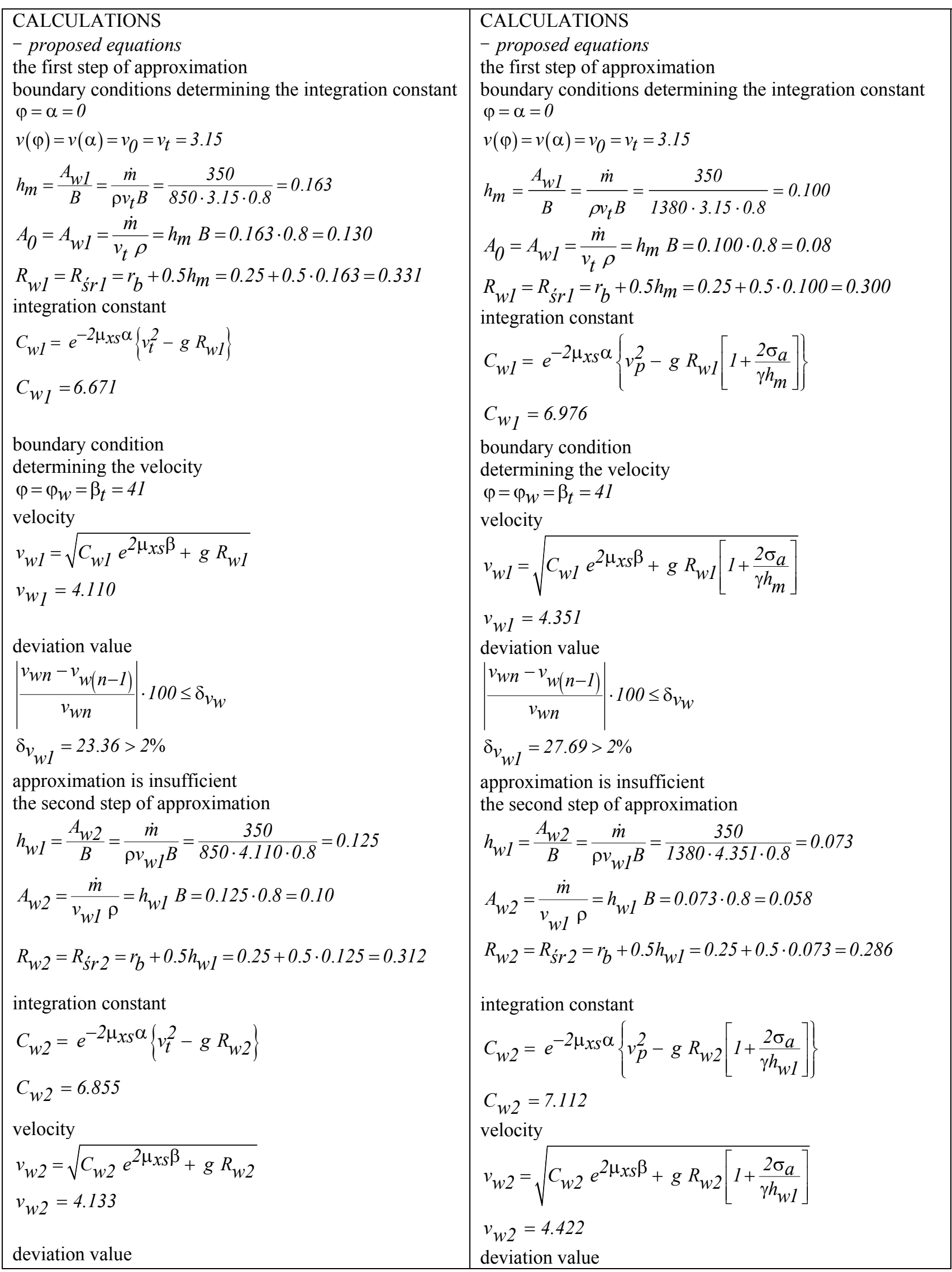




\begin{tabular}{|l|l|}
\hline$\delta_{v_{w 2}}=0.57<2 \%$ & $\delta_{v_{w 2}}=1.61<2 \%$ \\
- indirect equations & - indirect equations \\
$\beta_{t}=\alpha=0$ & $\beta_{t}=\alpha=0$ \\
$x_{0}=s_{0}+\left(R_{w 1}+0.5 \cdot h_{m}\right) \cdot \sin \beta_{t}$ & $x_{0}=s_{0}+\left(R_{w 1}+0.5 \cdot h_{m}\right) \cdot \sin \beta_{t}$ \\
$x_{0}=0.9$ & $x_{0}=0.9$ \\
$\xi_{0}=\operatorname{arctg}\left(\operatorname{tg} \beta_{t}-\frac{g \cdot x_{0}}{2 \cdot v_{w 1}^{2} \cdot \cos ^{2} \beta_{t}}\right)$ & $\xi_{0}=\operatorname{arctg}\left(\operatorname{tg} \beta_{t}-\frac{g \cdot x_{0}}{2 \cdot v_{w 1}^{2} \cdot \cos ^{2} \beta_{t}}\right)$ \\
$\xi_{0}=41$ & $\xi_{0}=41$ \\
$v_{0}=v_{t} \cdot \cos \beta_{t} \cdot \sqrt{\operatorname{tg}^{2} \xi_{0}+1}$ & $v_{0}=v_{t} \cdot \cos \beta_{t} \cdot \sqrt{\operatorname{tg}^{2} \xi_{0}+1}$ \\
$v_{0}=4.216$ & $v_{0}=4.216$ \\
- relative error of the determination of the velocity with & - relative error of the determination of the velocity with \\
both methods & both methods \\
$\delta_{v_{r}}=2 \%$ & $\delta_{v_{r}}=5 \%$ \\
\hline
\end{tabular}

The relative error $\delta_{v r}$ for results achieved with the proposed equations and with the indirect equations amounts to $2 \%$ for cohesionless materials and $5 \%$ for cohesive materials, respectively, which is acceptable for practical use. But the value of the error increases with the growing distance between the impact plate and head pulley. The difference between the velocity determined with the use of the indirect equations and the proposed equations for cohesionless materials amounts to $0.08[\mathrm{~m} / \mathrm{s}]$, and for cohesive materials to $0.21[\mathrm{~m} / \mathrm{s}]$. The minimal permissible distance between the throwing point and the impact plate was taken into consideration.

Differences obtained in calculations for values of velocity indicate that the proposed equations can be used for estimating the velocity of the material beyond the throwing point. The indirect equations take into consideration kinematic and geometric conditions, whereas the proposed equations take also into consideration dynamic parameters, adhesion, the coefficient of friction of a given material against the belt surface, as well as material parameters such as material bulk density and specific gravity.

\section{Conclusions}

The analysis of the conditions of flow of cohesionless and cohesive materials in a throwing point with a flat belt (non inclined conveyor) as well as the proposed equations obtained from the analysis are suitable for engineering calculations and for estimating the velocity of the material beyond the head pulley with the accuracy sufficient for practical use. It is especially important in cases when the value of the velocity beyond the point must be known. The knowledge of this value enables the correct selection of parameters of pouring points that cooperate with conveyors. It is also of importance for maintaining constant capacity of the transport system. The proposed equations consider not only kinematics and dynamics parameters of the flow but also material parameters.

The proposed equations, comparing to the indirect equations, give good compliance of $2 \%$ for cohesionless materials and 5\% for cohesive materials. Such value of the relative error was obtained for an impact plate operating in angular pouring points, whereas for parallel pouring points, where the distance between the head pulley and impact plate increases to $1.2 \mathrm{~m}$ (Żur [2]), the relative error can reach the value of almost $10 \%$.

The proposed equations provide a tool for engineers allowing estimating the value of velocity of the material beyond the throwing point, which assures the correct capacity of the transport system consisting of belt conveyors. The proposed equations prove to be useful in cases where a throwing point follows or precedes an impact point, especially at a properly selected distance between cooperating points, but they do not consider air resistance. 
The proposed equations assume that the angle at which the material falls onto the subsequent point is known. However, the equations may turn out to be useless for the assumed angle at which the material flows out of the head pulley if the distance is larger than $2 \mathrm{~m}$.

\section{Nomenclature}

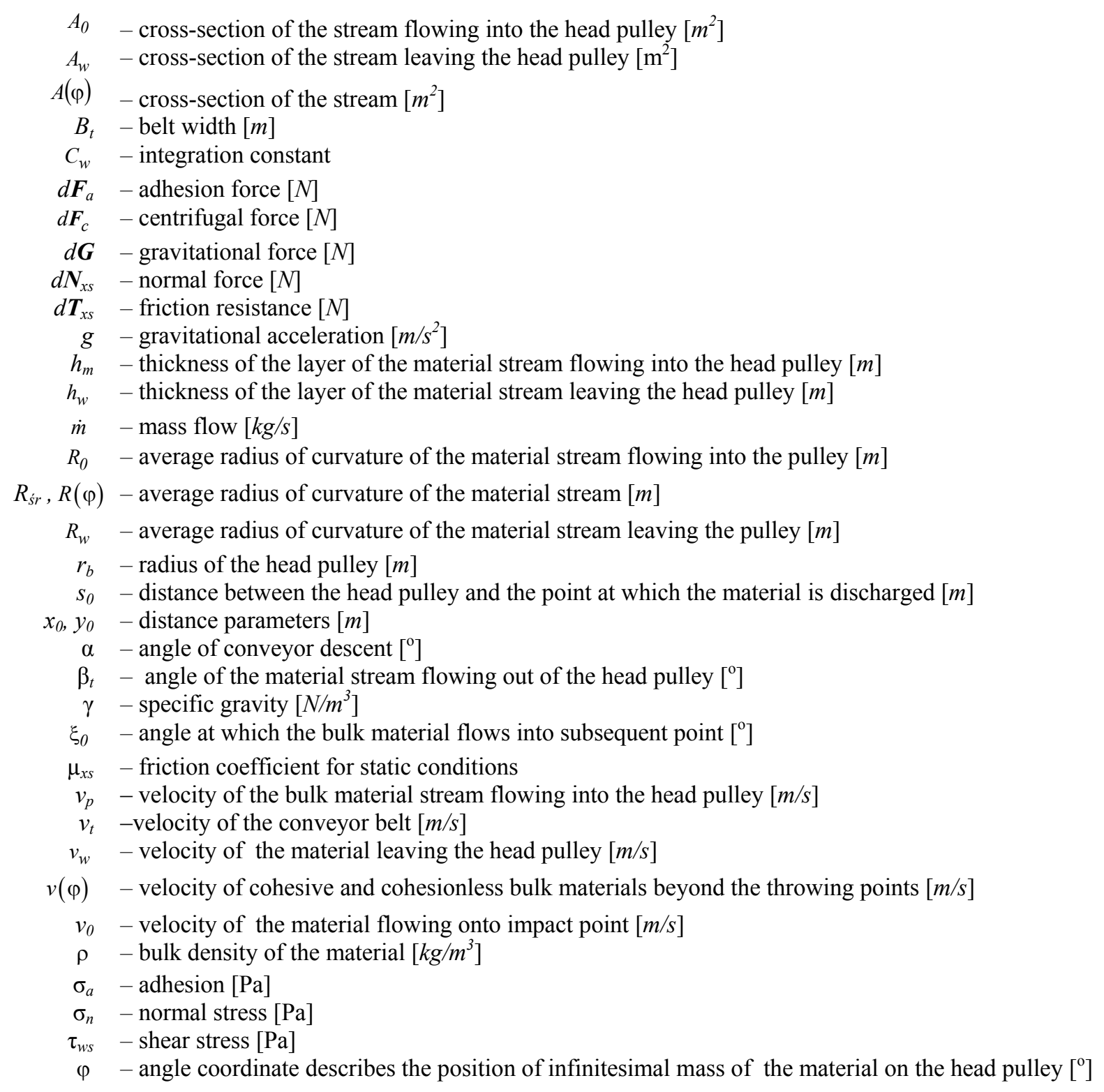

\section{References}

[1] Antoniak J. (2007a): Belt Conveyors in Underground and Opencast Mining. - Gliwice: Publishing House of the Silesian University of Technology.

[2] Żur T. (1979): Belt conveyors in mining. - Katowice: Publishing House Silesia.

[3] Cyganiuk J. (2013a): Safety of granular materials in conveyor transport systems, Machinery and equipment safety in industry. - Publishing Section of the Faculty of Management at the Częstochowa University of Technology, Częstochowa, pp.72-92. 
[4] Cyganiuk J. (2007c): Modelling of the flow of bulk material of group III in an impact pouring point with a flat impact plate. - Systems: Journal of Transdisciplinary Systems Science, vol.12, No.3 pp.67-76.

[5] Cyganiuk J. (2013b): Modelling of the flow of cohesionless bulk materials on a flat impact plates. - Surface Surface Mining, No.3-4 pp.39-45.

[6] Cyganiuk J. and Przystupa F.W. (2009): The influence of an inclination angle of the impact plate on the velocity of a material leaving the pouring point. - Surface Mining, No.4-5 pp.30-34.

[7] Korzeń Z. (1988): The dymanics of bulk solids flow on impact plates of belt conveyor systems. - Bulk Solid Handling, vol.8, No.6, pp.689-697.

[8] Przystupa F.W. and Cyganiuk J. (2007d): Modelling of the flow of fine material of a cement type in an impact pouring point with flat impact plate. - Surface Mining, No.3-4, pp.103-108.

[9] Sakowich W.L. and Kuksa W.P. (1968): Materials conveying in pouring devices of belt conveyors. - Machines for Construction of Mountain Roads, Tehnika, No.7, pp.78-86.

[10] Cyganiuk J. and Przystupa F. (2011): Modelling of the flow of cohesive bulk materials streams in throwing points with an ascending belt. - Surface Mining, No.3-4 pp.50-54.

[11] Cyganiuk J. (2007b): Modelling of the flow of bulk material streams in cooperating pouring points in conveying systems. - Surface Mining, No.3-4 pp.28-32.

[12] Cyganiuk J., Kuryło P. and Tertel E. (2014): Modelling of the flow of streams of cohesionless and cohesive bulk materials in a conveyor discharge point of a descending belt conveyor. - Procedia Engineering, No.96, pp.28-38.

[13] Korzeń Z. (1989): Mechanics of belt conveyor discharge process as affected by air drag. - Bulk Solids Handling, vol.9, No.3, pp.289-297.

[14] Korzeń Z. (1984): On the discharge processes of belt conveyors. - Fordern und Heben, vol.34, No.5, pp.380-388.

[15] Colijn H. (2011): Mechanical Conveyors for Bulk Solids. - Elsevier Science Publisher B.V., New York.

[16] Korzeń Z. (1990): Mechanics of bulk solid stream flow in throwing belt conveyors. - Bulk Solids Handling, vol.10, No.1, pp.55-64.

[17] Czuba W. and Furmanik K. (2013c): Analysis of a grain motion in the transfer area of the belt conveyor. Maintenance and Reliability, vol.15, No.4, pp.390-396.

[18] Antoniak J. (1970): Calculations of Conveyors Used in Mining. - Katowice: Publishing House Silesia.

[19] Arnold P.C. and Hill G.L. (1990): Predicting the discharge trajectory from belt conveyors. - Bulk Solids Handling, vol.10, No.4, pp.379-382.

[20] Hastie D. and Wypych P. (2010): Conveyor belt trajectories - comparing predicted to experimental results. Bulk Solids Handling, vol.30, No.8, pp.438-445.

Received: October 12, 2017

Revised: January 3, 2018 